\title{
Exploring Noise-Effect on the Intraband Transition Lifetime of Impurity Doped Quantum Dots
}

\author{
Sekh Md. Arif ${ }^{1}$ (D), Aindrila Bera ${ }^{1}$ (D), Anuja Ghosh ${ }^{1}$ (D), Manas Ghosh ${ }^{1, *}$ (D) \\ 1 Department of Chemistry, Physical Chemistry Section, Visva-Bharati University, Santiniketan, Birbhum 731 235, West \\ Bengal, India; arif8talpur@gmail.com (S.M.A.); aindrilabera1@gmail.com (A.B.); anuja.piya20@gmail.com (A.G.); \\ * Correspondence: pcmg77@ rediffmail.com;
}

Scopus Author ID 8258661600

Received: 27.06.2020; Revised: 28.07.2020; Accepted: 30.07.2020; Published: 2.08.2020

\begin{abstract}
Current inspection analyzes the role played by Gaussian white noise on intraband transition lifetime (ITL) of quantum dot $(Q D)$ containing an impurity. In this regard, the ITL profiles are scrutinized following the variation of several pertinent physical quantities with and without noise. Two different avenues viz. 'additive' and 'multiplicative' have been explored by which noise may be introduced to the system. Often, the presence of noise happens to alter the attributes of the ITL profiles (steady rise, steady fall, and maximization) from that under a noise-free state. And such alterations also prominently depend on the additive/multiplicative nature of noise. The present study clearly manifests the attainment of enhanced ITL of QDs containing dopants under applied Gaussian white additive noise having strength in the neighborhood of a typical value. The findings seem to have important bearings in designing and manufacturing QD-based optoelectronic devices where the role of noise needs to be acknowledged.
\end{abstract}

Keywords: quantum dot; impurity; Gaussian white noise; intraband transition lifetime; noise strength. (C) 2020 by the authors. This article is an open-access article distributed under the terms and conditions of the Creative Commons Attribution (CC BY) license (https://creativecommons.org/licenses/by/4.0/).

\section{Introduction}

Quantum dot $(Q D)$ is a renowned member of low-dimensional semiconductor systems (LDSS) family where the movement of electrons and holes suffers from complete spatial confinement. Above confinement has come out to be the driving force behind the exhibition of enhanced optical features of QD that are intimately linked with optical transitions, oscillator strengths, and light-matter interactions. In consequence, we have come across extensive application of QDs in various microelectronic and optoelectronic devices [1]. It is the effective confinement potential (ECP) of QD that actually guides the characteristics of optical properties. Thus, the fine-tuning of ECP of QD is of utmost relevance and can be accomplished by harnessing its shape, size, and composition.

QD containing dopant impurity is a well-known means to alter and tune its ECP. Such alteration and tuning affect the spatial distribution of QD energy levels, which, in turn, influences the various optical properties of QD. Quite naturally, there are a plethora of investigations involving dopant-related aspects in LDSS [2-18] with special focus on their nonlinear optical (NLO) properties [19-50]. The application of external noise is a viable route to modify the ECP of QD. The presence of noise invariably affects the output of devices where QD appears to be an essential ingredient. Hence it is absolutely pertinent to address the consequences of external noise on NLO properties of QD. 
The Quantum theory of radiation assumes that light is absorbed during the promotion of electrons to a higher energy level. However, photon emission takes place as the electron relaxes with a transition lifetime $(\tau)$ to a lower energy level and looses its excess energy. Thus, $\tau$ is the lifetime of the excited state before the spontaneous emission of the photon occurs. In other words, the emission corresponds to the process in which a photon with energy $\Delta E$ is released simultaneously, satisfying the conservation of energy requirement viz. $\Delta E=h v . \tau$ is influenced by various physical parameters such as pressure, temperature, electric field, and also by the size of the QD structure. The reciprocal of $\tau$ also bears some physical relevance as it denotes the total rate of spontaneous emission from a higher level to a lower level with the emission of a photon having energy $\Delta E=h v$ [51, 52]. Apart from being relevant in the fundamental physics of LDSS, $\tau$ also assumes importance from a technological perspective involving LDSS-based devices. $\tau$ affects the performance of these devices and may serve as a sensitive tool for assessing the processing cleanliness and material quality, thereby making the control of $\tau$ an important task [51]. We can, therefore, find several important works that deal with the control and modulation of $\tau$ in LDSS [51-55].

In the present work, we perform an extensive exploration of intraband transition lifetime (ITL, $\tau$ ) of doped QD for a transition between $\left|\Psi_{0}\right\rangle$ and $\left|\Psi_{1}\right\rangle$ states in light of the role played by Gaussian white noise. The present study envisions 2-d QD (GaAs) containing one electron and subject to lateral parabolic confinement such that the electronic motion becomes restricted to $x$ - $y$ plane. A magnetic field orthogonal to the $x-y$ plane is also applied. The doped impurity potential is described by a Gaussian function. Moreover, the doped QD is subjected to an external static electric field $(F)$ applied through $x$ and $y$ axes. Two different pathways, namely additive and multiplicative, have been invoked for the entry of noise into the system. Based on the mode of entry, noise interacts differently with the system leading to diverse features in the NLO properties, which are not observed under a noise-free state. The Present work examines the features of ITL profiles following the variations of several important physical parameters such as electric field $(F)$, magnetic field $(B)$, confinement potential $\left(\omega_{0}\right)$, dopant location $\left(r_{0}\right)$, dopant potential $\left(V_{0}\right)$, binding energy (BE), aluminium concentration (y) (considering doped $A l_{y} G a_{1-y} A s$ QD) [19], noise strength ( $\zeta$ ), positiondependent effective mass (PDEM) [56-64], position-dependent dielectric screening function (PDDSF) [56, 58, 65], geometrical anisotropy [66-69], hydrostatic pressure (HP) [6, 8, 23, 25, 33, 36, 70, 71] and temperature (T) [70, 71]. The study elucidates the impact of noise in fabricating the ITL profiles of doped QD when different physical quantities undergo progressive change.

\section{Materials and Methods}

The complete Hamiltonian representing the system, taking into cognizance the dopant (impurity), the electric field and noise, may be written as

$$
H_{0}=H_{0}^{\prime}+V_{\text {imp }}+|e| F(x+y)+\xi(x, y)
$$

Invoking effective mass approximation, it seems that $H_{0}^{\prime}$ stands for impurity-free, single-electron QD where the electronic motion is restricted to the $x-y$ plane owing to lateral parabolic confinement $V(x, y)=\frac{1}{2} m^{*} \omega_{0}^{2}\left(x^{2}+y^{2}\right) . \quad \omega_{0}$ can be viewed as the harmonic confinement frequency and $m^{*}$ is the effective mass of an electron. $H_{0}^{\prime}$ can be given by 


$$
H_{0}^{\prime}=\frac{1}{2 m^{*}}\left[-i \hbar \nabla+\frac{e}{c} \overrightarrow{\mathrm{A}}\right]^{2}+\frac{1}{2} m^{*} \omega_{0}^{2}\left(x^{2}+y^{2}\right)
$$

The perpendicular magnetic field $(B)$ makes its presence in the above equation through the expression $\omega_{c}=\frac{e B}{m^{*} c}$ using Landau gauge $[A=(B y, 0,0)], \vec{A}$ being the vector potential. $\omega_{c}$ is the cyclotron frequency which can be regarded as manifesting the role of the magnetic field. Above Hamiltonian can also be written as

$$
H_{0}^{\prime}=-\frac{\hbar^{2}}{2 m^{*}}\left(\frac{\partial^{2}}{\partial x^{2}}+\frac{\partial^{2}}{\partial y^{2}}\right)+\frac{1}{2} m^{*} \omega_{0}^{2} x^{2}+\frac{1}{2} m^{*} \Omega^{2} y^{2}-i \hbar \omega_{c} y \frac{\partial}{\partial x} .
$$

Where, $\Omega\left(=\sqrt{\omega_{0}^{2}+\omega_{c}^{2}}\right)$ is the overall confinement frequency in the $y$-direction.

The impurity (dopant) potential $V_{\text {imp }}$ of eqn. (1) is delineated by a Gaussian function viz. $V_{\text {imp }}=V_{0} e^{-\gamma\left[\left(x-x_{0}\right)^{2}+\left(y-y_{0}\right)^{2}\right]}$. It comprises three major dopant parameters, namely $\left(x_{0}, y_{0}\right)$, $V_{0}$, and $\gamma$. They stand for the location of dopant incorporation, the dopant potential and a measure of the spatial extension of impurity effect, respectively. $\gamma$ is related to the dielectric constant $(\varepsilon)$ of the medium.

$\xi(x, y)$ of eqn. (1) takes care of noise characterized by zero-average and spatial $\delta$ correlation. We introduce a function $g(x, y)$ which mathematically describes above mentioned physical characteristics of noise such that

$$
\langle g(x, y)\rangle=0
$$

and

$$
\left\langle g(x, y) g\left(x^{\prime}, y^{\prime}\right)\right\rangle=2 \zeta \delta\left[(x, y)-\left(x^{\prime}, y^{\prime}\right)\right]
$$

respectively, where $\zeta$ is the noise strength. The function $g(x, y)$ has been produced by invoking the Box-Muller algorithm and $g(x, y)$ follows a Gaussian distribution. $\xi(x, y)$ is called 'additive' noise or 'multiplicative' noise based on its mode of interactions with the system. To be precise, for additive white noise (AWN) we write

$$
\xi(x, y)=\lambda_{1} g(x, y)
$$

And with multiplicative white noise (MWN) it becomes

$$
\xi(x, y)=\lambda_{2} g(x, y)(x+y),
$$

where $\lambda_{1}$ and $\lambda_{2}$ are two arbitrary parameters.

The time-independent Schrödinger equation has been solved using a variational recipe. The trial function $\left[\psi_{k}(x, y)\right]$ has been expressed as a linear combination of product of harmonic oscillator eigenfunctions $\phi_{n}(p x)$ and $\phi_{m}(q y)$ so that

$$
\psi_{k}(x, y)=\sum_{n m} C_{n m, k} \phi_{n}(p x) \phi_{m}(q y)
$$

where $C_{n m, k}$ are the linear combination coefficients and $p=\sqrt{\frac{m^{*} \omega_{0}}{\hbar}}$ and $q=\sqrt{\frac{m^{*} \Omega}{\hbar}}$. The matrix elements of various terms of eqn(1) have been calculated using the above trial function.

Calculation of said matrix elements helps us construct the Hamiltonian matrix for $H_{0}$ [cf. eqn. (1)] followed by its diagonalization leading to the attainment of the energy levels and the normalized eigenfunctions. 
Following the density matrix formalism, $\tau$ between the ground and the first excited state is given by $[54,72,73]$

$$
\tau=\frac{3 c^{3} h^{4} \varepsilon_{0}}{16 \pi^{3} \Delta E_{12}^{3} n_{r}\left|M_{12}\right|^{2}},
$$

where $c, h, \varepsilon 0, \Delta E_{12}, n_{r}$ and $M_{12}$ are the speed of light in vacuum, Planck's constant, vacuum permittivity, transition energy between the ground state and the first excited state, refractive index, and the transition dipole moment integral between the ground state and the first excited state, respectively.

\section{Results and Discussion}

In the present work, the values of dielectric constant and electronic effective mass are chosen to be $\varepsilon=12.4, m^{*}=0.067 m_{0}$, respectively, where $m_{0}$ is the electronic mass in a vacuum. The above values of $\varepsilon$ and $m^{*}$ are suitable for GaAs QDs. Unless otherwise mentioned, throughout the study we have used $\hbar \omega_{0}=250.0 \mathrm{meV}, F=100 \mathrm{kV} / \mathrm{cm}, B=10.0 \mathrm{~T}, V_{0}=280.0$ $\mathrm{meV}, r_{0}=0.0 \mathrm{~nm}$ and $\zeta=1.0 \times 10^{-4}$. In all the plots discussed below, the ITL profiles are inspected as various physical parameters vary over a range under noise-free conditions (i) and in the presence of AWN (ii) and MWN (iii), respectively. And the physical explanations behind various ITL profiles have been given after describing all the plots.

\subsection{Role of the electric field $(F)$.}

Fig. 1 depicts the ITL profiles as a function of electric field strength. Both without noise and with MWN, ITL displays steady enhancement with $F$. However, the outcome changes in the presence of AWN whence ITL displays prominent maximization at $F \sim 70 \mathrm{kV} / \mathrm{cm}$.

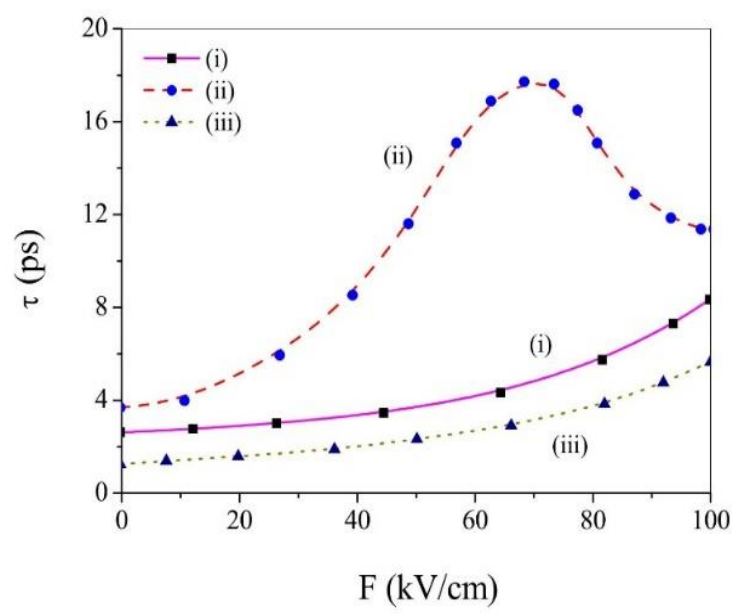

Figure 1. Plots of ITL vs $F$ : (i) under noise-free condition, (ii) in the presence of AWN, and (iii) with MWN.

\subsection{Role of the magnetic field (B).}

Fig. 2 delineates the ITL profile as a function of magnetic field strength. The ITL profiles display a regular increase with an increase in $B$ without noise and with MWN. However, under applied AWN, ITL decreases steadily as $B$ enhances and finally saturates at large $B$. 


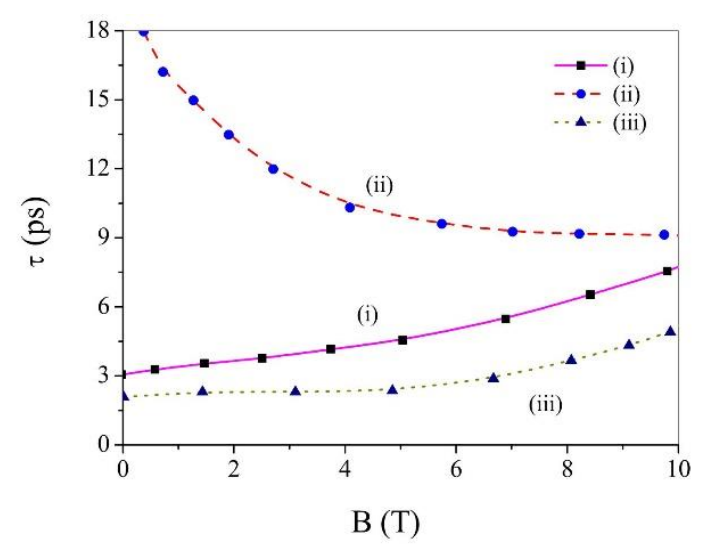

Figure 2. Plots of ITL vs. $B$ : (i) under noise-free condition, (ii) in the presence of AWN, and (iii) with MWN.

\subsection{Role of confinement frequency $\left(\omega_{0}\right)$.}

Fig. 3 exhibits the ITL profiles following the variation of confinement energy $h \omega_{0}$. The plots divulge the emergence of maximization without noise and under applied AWN at $h \omega_{0} \sim$ $70 \mathrm{meV}$ and at $h \omega_{0} \sim 80 \mathrm{meV}$, respectively. In contrast, under applied MWN, the ITL plot reveals a feeble minimization at $h \omega_{0} \sim 30 \mathrm{meV}$, followed by steady enhancement with further increase in $h \omega_{0}$.

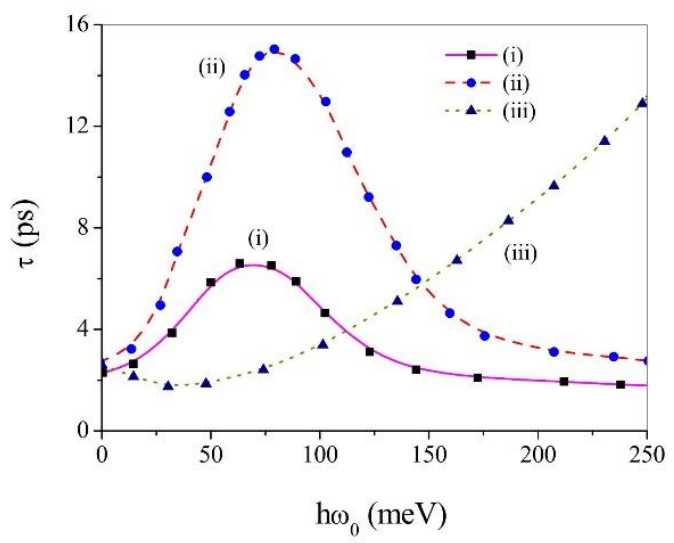

Figure 3. Plots of ITL vs $h \omega_{0}$ : (i) under noise-free condition, (ii) in the presence of AWN, and (iii) with MWN.

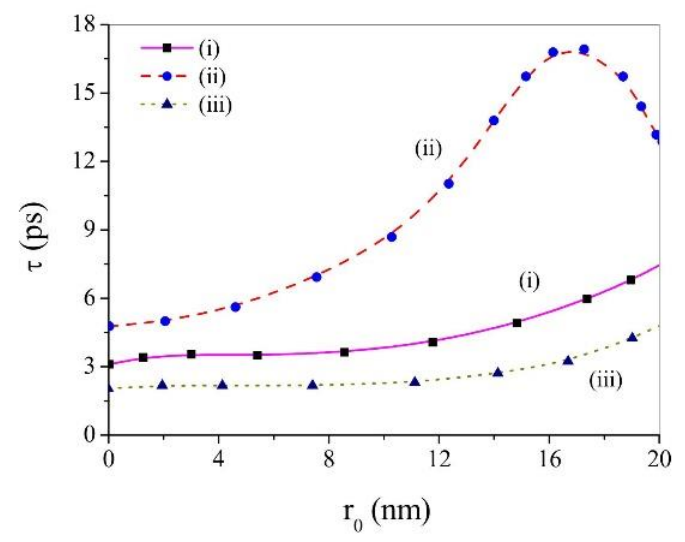

Figure 4. Plots of ITL vs. $r_{0}$ : (i) under noise-free condition, (ii) in the presence of AWN, and (iii) with MWN. 


\subsection{Role of dopant location ( $\left.r_{0}\right)$.}

Fig. 4 depicts the variation of ITL profiles as a function of dopant location. The profiles appear quite similar without noise and in the presence of MWN as in both the cases, the said profiles show a steady increase with an increase in $r_{0}$, i.e., with the gradual shift of dopant from on-center to more and more off-center locations. However, in the presence of AWN the ITL profile exhibits maximization around $r_{0} \sim 17 \mathrm{~nm}$.

\subsection{Role of dopant potential $\left(V_{0}\right)$.}

Fig. 5 delineates the variation of ITL with an increase in the strength of the dopant potential. This plot carries specific importance as we can monitor how ITL changes as we proceed from QD without any impurity to QD infused with a certain amount of impurity. The plot reveals that, regardless of the presence/absence of noise, ITL undergoes steady enhancement with an increase in $V_{0}$.

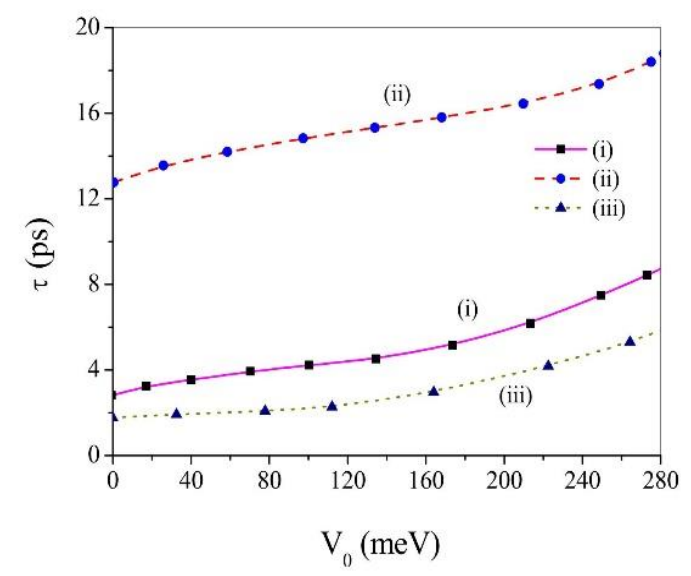

Figure 5. Plots of ITL vs. $V_{0}$ : (i) under noise-free condition, (ii) in the presence of AWN, and (iii) with MWN.

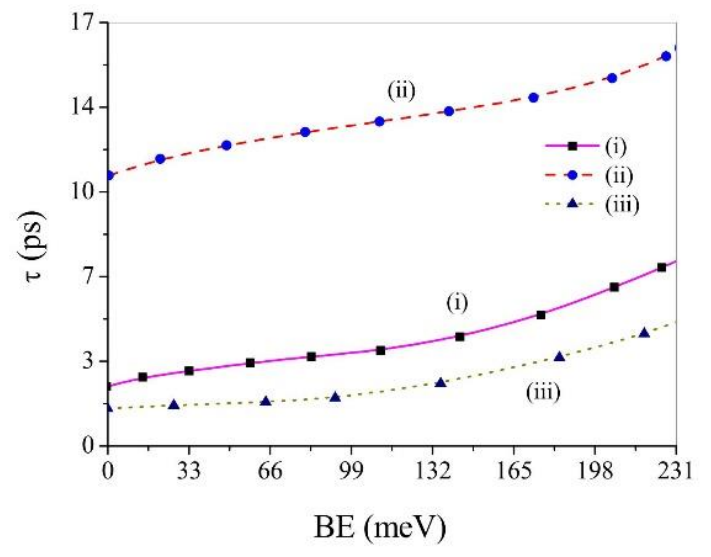

Figure 6. Plots of ITL vs. BE: (i) under noise-free condition, (ii) in the presence of AWN, and (iii) with MWN.

3.6. Role of binding energy $(B E)$.

The ground state binding energy $E_{B}$ may be represented as

$$
E_{B}=E_{0}-E,
$$

Where $E$ and $E_{0}$ are the ground state energies of QD containing impurity and dopant-free QD, respectively. Fig. 6 depicts the ITL profiles as a function of BE of the system. The findings are 
considered analogous to that of the previous ITL vs. Vo plots [fig. 5]. We, therefore, abstain from elaborating these plots.

\subsection{Role of aluminum concentration (y).}

We now examine the role of aluminum concentration $(y)$ on ITL profiles upon considering $A l_{y} G a_{1-y} A s$ QD system whose effective mass is given by $m^{*}=(0.067+0.083 y) m_{0}$ [19]. Fig. 7 exhibits the pattern of variation of ITL profiles with $y$. The ITL vs. $y$ profiles exhibit a similar trend under noise-free state, and with AWN as in both the cases, we find a regular increase in ITL as $y$ increases. However, under applied MWN, ITL displays noticeable maximization at $y \sim 0.6$.

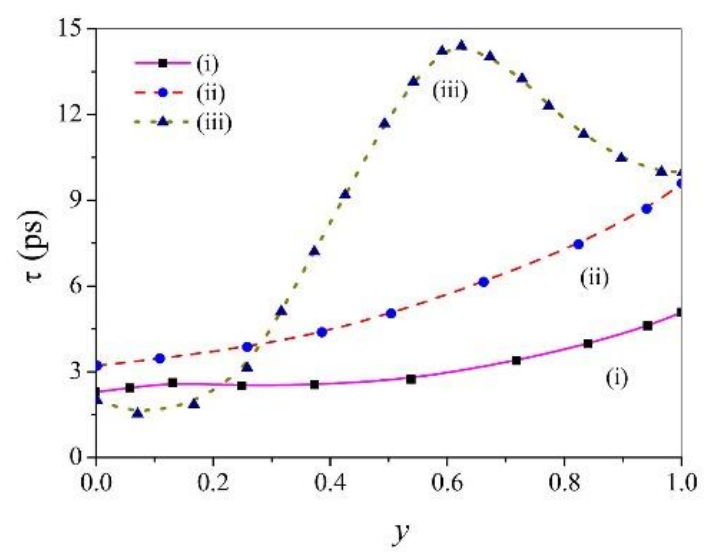

Figure 7. Plots of ITL vs. $y$ : (i) under noise-free condition, (ii) in the presence of AWN, and (iii) with MWN.

\subsection{Role of the position-dependent effective mass (PDEM).}

The effective mass becomes dependent on the dopant site $\left(r_{0}\right)$ in the case of PDEM. The present study involves the expressions of PDEM given in the studies done by Rajashabala and Navaneethakrishnan [56] and Peter and Navaneethakrishnan [58]. Fig. 8 presents the ITL profiles following the variation of $m^{*}\left(r_{0}\right)$. The ITL profiles display a similar trend under noisefree state and with MWN. In both these cases, ITL manifests a moderate increase as $m^{*}\left(r_{0}\right)$ increases. However, under the influence of AWN, the said profile exhibits maximization at $m^{*}\left(r_{0}\right) \sim 0.6$.

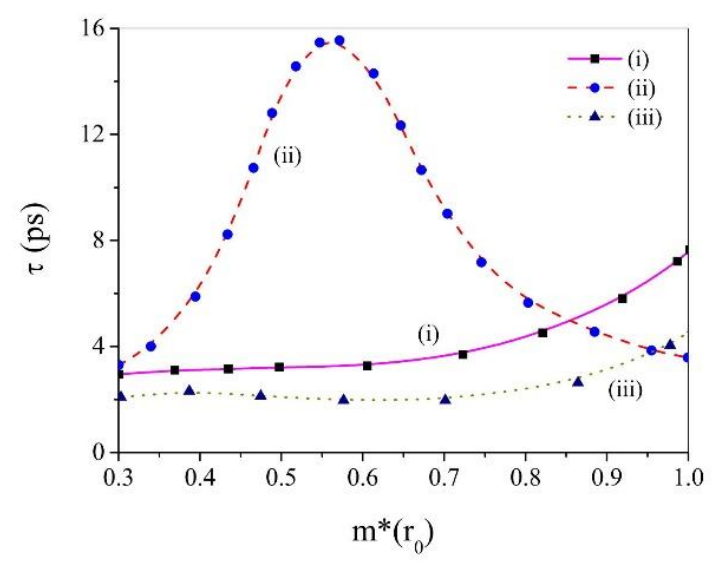

Figure 8. Plots of ITL vs. $m^{*}\left(r_{0}\right):$ (i) under noise-free condition, (ii) in the presence of AWN, and (iii) with MWN. 
3.9. Role of position-dependent dielectric screening function (PDDSF).

Similar to PDEM, in this case, the dielectric screening function becomes dependent on the dopant coordinate. The current investigation explores Hermanson's PDDSF, as discussed in the studies made by Rajashabala and Navaneethakrishnan [56], Peter and Navaneethakrishnan [58] and Deng et al. [65]. Fig. 9 depicts the ITL profiles against PDDSF denoted by $\varepsilon\left(r_{0}\right)$ under different conditions. It is observed that irrespective of the presence of noise, ITL shows noticeable enhancement with an increase in $\varepsilon\left(r_{0}\right)$. The enhancement appears to be most prominent in the presence of AWN.

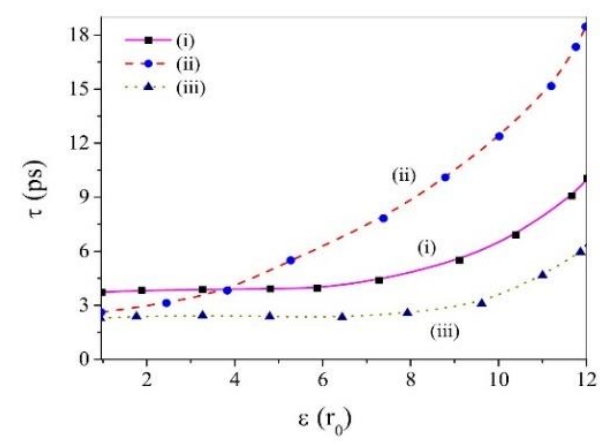

Figure 9. Plots of ITL vs. $\varepsilon\left(r_{0}\right)$ : (i) under noise-free condition, (ii) in the presence of AWN, and (iii) with MWN.

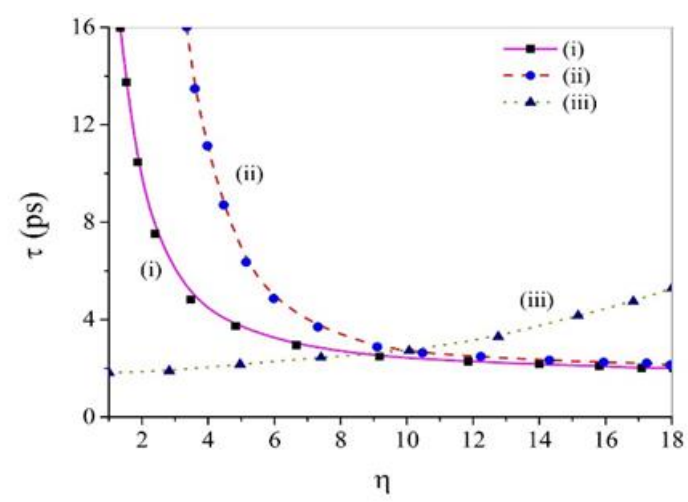

Figure 10. Plots of ITL vs. $\eta$ : (i) under noise-free condition, (ii) in the presence of AWN, and (iii) with MWN.

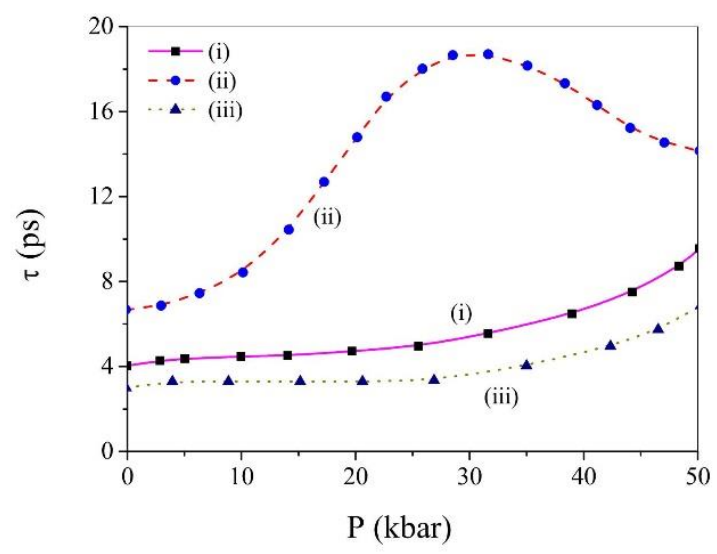

Figure 11. Plots of ITL vs. hydrostatic pressure: (i) under noise-free condition, (ii) in the presence of AWN, and (iii) with MWN. 
3.10. Role of geometrical anisotropy $(\eta)$.

As per Xie, the quantity $\eta=\frac{\Omega}{\omega_{0}}$ [cf. eqn (3)] could be viewed as the geometrical anisotropy parameter [66, 67]. Fig. 10 evinces the ITL profiles against $\eta$ under different conditions. Without noise and under the aegis of AWN, the ITL profiles display a steady decline with an increase in $\eta$. On the other hand, in the presence of MWN, the said profile reveals a gentle rise with an increase in $\eta$.

\subsection{Role of hydrostatic pressure (HP).}

The role of HP and temperature can be included upon considering the pressure and temperature-dependence of $m^{*}$ and $\varepsilon$. For GaAs QD these dependencies are given in the work of Lu et al. [71]. Fig. 11 shows the ITL profiles with a change of HP under various conditions. The ITL profiles exhibit moderate enhancement with an increase in pressure without noise [54] and under MWN. In the presence of AWN, the ITL profile displays noticeable maximization at $\mathrm{P} \sim 30$ kbar.

\subsection{Role of temperature $(T)$.}

Fig. 12 demonstrates the ITL profiles as temperature varies over a range. In this case, we can clearly distinguish the trend of the said profiles without and with noise. Whereas without noise, the ITL profile reveals a gentle drop with an increase in temperature [54], the trend exactly reverses in the presence of noise regardless of its mode of application. In the presence of noise, the ITL profiles exhibit persistent enhancement with an increase in temperature.

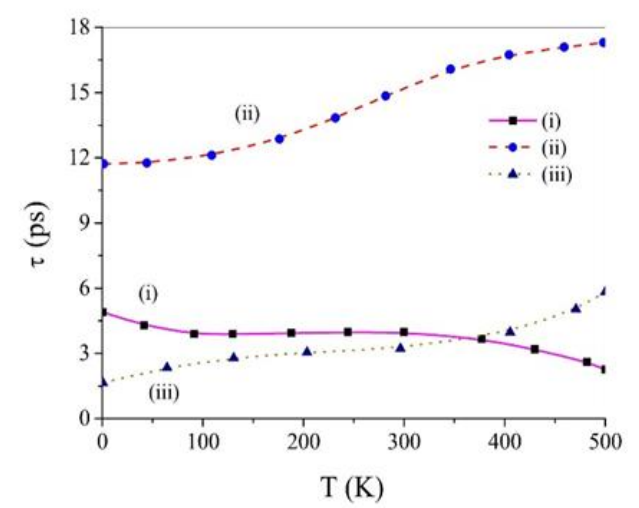

Figure 12. Plots of ITL vs. temperature: (i) under noise-free condition, (ii) in the presence of AWN, and (iii) with MWN.

\subsection{Possible explanation of observed ITL profiles.}

Different patterns of ITL profiles under different atmospheres can be explained on the basis of the product $\Delta E_{12}^{3}\left|M_{12}\right|^{2}$ present in its defining equation [cf. eqn (9)] [54]. The ITL profiles delineated so far (when different physical parameters undergo steady variation) have exhibited steady growth, steady decline, maximization, and saturation. These features depend upon the given physical quantity involved, the range over which it varies, existence/nonexistence of noise, and obviously the noise-mode (additive/multiplicative). An increase 
(decrease) in the above product will reduce (enhance) the ITL. The term $\left|M_{12}\right|$ of the product will be large (small) if there is a substantial (marginal) overlap between the concerned eigenstates. On the other hand, a large (small) energy separation between the eigenstates will make $\Delta E_{12}$ large (small). However, both of these two quantities may or may not increase/decrease simultaneously as different physical quantities are varied under various ambiances. Thus, the change in the overall magnitude of the above product is not at all straightforward. Depending upon the particular physical quantity undergoing variation, presence/absence of noise and the choice of the noise mode (if noise is present), the aforesaid product may decrease steadily, may increase steadily, may be minimized or may itself remain steady leading to steady rise, steady fall, maximization and saturation in the ITL profiles, respectively. For convenience, the results found are summarized in Table 1.

Table 1. ITL profiles during variations of different physical quantities.

\begin{tabular}{|c|c|c|c|}
\hline Sl. No. & $\begin{array}{c}\text { Physical parameter being } \\
\text { varied }\end{array}$ & Condition & $\begin{array}{l}\text { Behavior of } \tau \text { with an increase in the } \\
\text { physical parameters }\end{array}$ \\
\hline 1. & $F$ & $\begin{array}{c}\text { noise-free } \\
\text { additive noise } \\
\text { multiplicative noise }\end{array}$ & $\begin{array}{c}\text { increases } \\
\text { maximizes at } F \sim 70 \mathrm{kV} / \mathrm{cm} \\
\text { increases }\end{array}$ \\
\hline 2. & $B$ & $\begin{array}{c}\text { noise-free } \\
\text { additive noise } \\
\text { multiplicative noise } \\
\end{array}$ & $\begin{array}{l}\text { increases } \\
\text { decreases } \\
\text { increases }\end{array}$ \\
\hline 3. & $\omega_{0}$ & $\begin{array}{c}\text { noise-free } \\
\text { additive noise } \\
\text { multiplicative noise }\end{array}$ & $\begin{array}{c}\text { maximizes at } h \omega_{0} \sim 70 \mathrm{meV} \\
\text { maximizes at } h \omega_{0} \sim 80 \mathrm{meV} \\
\text { increases }\end{array}$ \\
\hline 4. & $r_{0}$ & $\begin{array}{c}\text { noise-free } \\
\text { additive noise } \\
\text { multiplicative noise }\end{array}$ & $\begin{array}{c}\text { increases } \\
\text { maximizes at } r_{0} \sim 17 \mathrm{~nm} \\
\text { increases }\end{array}$ \\
\hline 5. & $V_{0}$ & $\begin{array}{c}\text { noise-free } \\
\text { additive noise } \\
\text { multiplicative noise } \\
\end{array}$ & $\begin{array}{c}\text { increases } \\
\text { do } \\
\text { do } \\
\end{array}$ \\
\hline 6. & $B E$ & $\begin{array}{c}\text { noise-free } \\
\text { additive noise } \\
\text { multiplicative noise } \\
\end{array}$ & $\begin{array}{c}\text { increases } \\
\text { do } \\
\text { do } \\
\end{array}$ \\
\hline 7. & $y$ & $\begin{array}{c}\text { noise-free } \\
\text { additive noise } \\
\text { multiplicative noise }\end{array}$ & $\begin{array}{c}\text { increases } \\
\text { do } \\
\text { maximizes at } y \sim 0.6\end{array}$ \\
\hline 8. & PDEM & $\begin{array}{c}\text { noise-free } \\
\text { additive noise } \\
\text { multiplicative noise }\end{array}$ & $\begin{array}{c}\text { increases } \\
\text { maximizes at } m^{*}\left(r_{0}\right) \sim 0.6 \\
\text { increases }\end{array}$ \\
\hline 9. & $P D D S F$ & $\begin{array}{c}\text { noise-free } \\
\text { additive noise } \\
\text { multiplicative noise } \\
\end{array}$ & $\begin{array}{c}\text { increases } \\
\text { do } \\
\text { do } \\
\end{array}$ \\
\hline 10. & $\eta$ & $\begin{array}{c}\text { noise-free } \\
\text { additive noise } \\
\text { multiplicative noise } \\
\end{array}$ & $\begin{array}{l}\text { decreases } \\
\text { do } \\
\text { increases }\end{array}$ \\
\hline 11. & $P$ & $\begin{array}{c}\text { noise-free } \\
\text { additive noise } \\
\text { multiplicative noise }\end{array}$ & $\begin{array}{c}\text { increases } \\
\text { maximizes at } P \sim 30 \mathrm{kbar} \\
\text { increases }\end{array}$ \\
\hline 12. & $T$ & $\begin{array}{c}\text { noise-free } \\
\text { additive noise } \\
\text { multiplicative noise }\end{array}$ & $\begin{array}{l}\text { decreases } \\
\text { increases } \\
\text { increases }\end{array}$ \\
\hline
\end{tabular}

\subsection{Role of noise strength ( $\zeta)$.}

We now inspect the role of noise strength on the ITL profiles. Fig. 13 describes ITL profiles against $-\log (\zeta)$ both under applied AWN and MWN, respectively. The plot reveals the distinct maximization of ITL at $\zeta \sim 10^{-9}$ under applied AWN. However, in the presence of applied MWN, the ITL profile exhibits an overall decreasing trend with an increase in the noise 
strength consisting of gentle minimization and maximization at $\zeta \sim 10^{-7.5}$ and $\zeta \sim 10^{-4}$, respectively. It goes without saying that for vanishingly small values of noise strength, the ITL approaches its noise-free limit both under applied AWN and MWN. Thus, the application of AWN of strength $\zeta \sim 10^{-9}$ seems to be conducive for the achievement of a large intraband transition lifetime of doped QD.

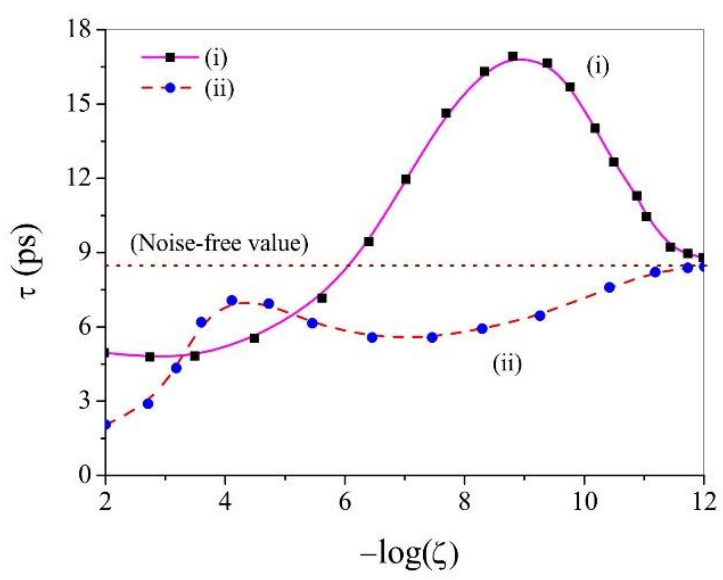

Figure 13. Plots of ITL vs. $-\log (\zeta)$ : (i) in the presence of AWN and (ii) in the presence of MWN.

\section{Conclusions}

Intraband transition lifetime (ITL) of QD contaminated with dopant has been examined in conjunction with the active presence of Gaussian white noise. Two different routes (additive and multiplicative) have been conceived through which said noise could ingress the system. And the influence of noise has been carefully and delicately monitored as several significant physical quantities are varied. On several occasions, the presence of noise alters the features of the ITL profiles from that under a noise-free state. Furthermore, the aforesaid additive and multiplicative nature of noise also bring about diversities in the ITL profiles. The ITL profiles comprise of important features like steady rise, steady fall, and maximization that heavily depend on the presence of noise and its mode of application. On the whole, the present study proclaims the achievement of large ITL of doped QDs in the presence of Gaussian white additive noise having strength in the vicinity of $\zeta \sim 10^{-9}$. The above findings may serve as prerequisites for the fabrication of optoelectronic devices containing QD as an essential component where the role of noise must be duly acknowledged.

\section{Funding}

This research received no external funding.

\section{Acknowledgments}

The authors S. M. A., A. B., A. G., and M. G. thank DST-FIST (Government of India) and UGC-SAP (Government of India) for support.

\section{Conflicts of Interest}

The authors declare no conflict of interest. 


\section{References}

1. Yakar, Y.; Çakir, B.; Özmen, A. Magnetic field effects on oscillator strength, dipole polarizability and refractive index changes in spherical quantum dot. Chem. Phys. Lett. 2018, 708, 138-145, https://doi.org/10.1016/j.cplett.2018.08.010.

2. Xie, W. Impurity effects on optical property of a spherical quantum dot in the presence of an electric field. Physica B 2010, 405, 3436-3440, https://doi.org/10.1016/j.physb.2010.05.019.

3. Khordad, R.; Bahramiyan, H. Impurity position effect on optical properties of various quantum dots. Physica E 2015, 66, 107-115, https://doi.org/10.1016/j.physe.2014.09.021.

4. Baskoutas, S.; Paspalakis, E.; Terzis, A.F. Electronic structure and nonlinear optical rectification in a quantum dot: effects of impurities and external electric field. J. Phys.: Condensed Matter 2007, 19.

5. Niculescu, E.C.; Stan, C.; Cristea, M.; Truscă, C. Magnetic-field dependence of the impurity states in a dome-shaped quantum dot. Chem. Phys. 2017, 493, 32-41, https://doi.org/10.1016/j.chemphys.2017.06.004.

6. Ghazi, H.E.; Jorio, A.; Zorkani, I. Pressure-dependent shallow donor binding energy in InGaN/GaN square QWWs. Physica B 2013, 410, 49-52.

7. Stevanović, L.; Filipović, N.; Pavlović, V. Effect of magnetic field on absorption coefficients, refractive index changes and group index of spherical quantum dot with hydrogenic impurity. Optical Materials 2019, 91, 62-69, https://doi.org/10.1016/j.optmat.2019.02.049.

8. Akbas, H.; Erdogan, I.; Akankan, O. Hydrostatic pressure effects on impurity states in GaAs/AlAs quantum wells. Superlattices and Microstructures 2011, 50, 80-89, https://doi.org/10.1016/j.spmi.2011.05.006.

9. Akbas, H.; Dane, C.; Erdogan, I.; Akankan, O. Hydrogenic donor in asymmetric quantum wells. Physica E 2014, 60, 196-199, https://doi.org/10.1016/j.physe.2014.03.003.

10. Boz, F.K.; Aktas, S.; Bilekkaya, A.; Okan, S.E. The multilayered spherical quantum dot under a magnetic field. Appl. Surf. Sci. 2010, 256, 3832-3836, https://doi.org/10.1016/j.apsusc.2010.01.036.

11. Cristea, M. Simultaneous effects of electric field, shallow donor impurity and geometric shape on the electronic states in ellipsoidal $\mathrm{ZnS} / \mathrm{CdSe}$ core-shell quantum dots. Physica E 2018, 103, 300-306, https://doi.org/10.1016/j.physe.2018.06.019.

12. Yadri, M.E.; Aghoutane, N.; Aouami, A.E.; Feddi, E.; Dujardin, F.; Duque, C.A. Temperature and hydrostatic pressure effects on single dopant states in hollow cylindrical core-shell quantum dot. Applied Surface Science 2018, 441, 204-209, https://doi.org/10.1016/j.apsusc.2018.01.195.

13. Barseghyan, M.G.; Mughnetsyan, V.N.; Pérez, L.M.; Kirakosyan, A.A.; Laroze, D. Effect of the impurity on the Aharonov-Bohm oscillations and the intraband absorption in $\mathrm{GaAs} / \mathrm{Ga}_{1-\mathrm{x}} \mathrm{Al}_{\mathrm{x}} \mathrm{As}$ quantum ring under intense THz laser field. Physica E 2019, 111, 91-97, https://doi.org/10.1016/j.physe.2019.03.003.

14. Shi, L.; Yan, Z.W. Stark Shift and photoionization cross section of on-center and off-center donor impurity in a core/shell ellipsoidal quantum dot. Physica E 2018, 98, 111-117, https://doi.org/10.1016/j.physe.2017.12.034.

15. Holovatsky, V.A.; Voitsekhivska, O.M.; Yakhnevych, M.Y. The effect of magnetic field and donor impurity on electron spectrum in spherical core-shell quantum dot. Superlattices and Microstructures 2018, 116, 916, https://doi.org/10.1016/j.spmi.2018.02.006.

16. Bejan, D.; Stan, C.; Niculescu, E.C. Effects of electric field and light polarization on the electromagnetically induced transparency in an impurity doped quantum ring. Optical Materials 2018, 75, 827-840, https://doi.org/10.1016/j.optmat.2017.11.047.

17. Hosseinpour, P. The role of Rashba spin-orbit interaction and external fields in the thermal properties of a doped quantum dot with Gaussian impurity. Physica B 2020, 593, 412259, https://doi.org/10.1016/j.physb.2020.412259.

18. Safwan, S.A.; Meshad, N.E.; Saleh, A.; Hassanein, H.M. Stark shift of hydrogenic and non-hydrogenic donor impurity excited states in parabolic quantum dot: Under the effect of electric field and temperature. Physica E 2020, 118, 113882, https://doi.org/10.1016/j.physe.2019.113882.

19. Rezaei, G.; Vahdani, M.R.K.; Vaseghi, B. Nonlinear optical properties of a hydrogenic impurity in an ellipsoidal finite potential quantum dot. Current Appl. Phys. 2011, 11, 176-181, https://doi.org/10.1016/j.cap.2010.07.002.

20. Rezaei, G.; Vaseghi, B.; Taghizadeh, F.; Vahdani, M.R.K.; Karimi, M.J. Intersubband optical absorption coefficient changes and refractive index changes in a two-dimensional quantum pseudodot system. Superlattices and Microstructures 2010, 48, 450-457, https://doi.org/10.1016/j.spmi.2010.08.009.

21. Lu, L.; Xie, W.; Hassanabadi, H. Linear and nonlinear optical absorption coefficients and refractive index changes in a two-electron quantum dot. J. Appl. Phys. 2011, 109, https://doi.org/10.1063/1.3560541.

22. Karabulut, I.; Atav, Ü.; Şafak, H.; Tomak, M. Linear and nonlinear intersubband optical absorptions in an asymmetric rectangular quantum well. Euro. Phys. J. B 2007, 55, 283-288, https://doi.org/10.1140/epjb/e2007-00055-1.

23. Karabulut, I.; Atav, Ü.; Şafak, H.; Tomak, M. Second harmonic generation in an asymmetric rectangular quantum well under hydrostatic pressure. Physica B 2007, 393, 133-138, https://doi.org/10.1016/j.physb.2006.12.074. 
24. Kasapoglu, E.; Ungan, F.; Sari, H.; Sökmen, I.; Mora-Ramos, M.E.; Duque, C.A. Donor impurity states and related optical responses in triangular quantum dots under applied electric field. Superlattices and Microstructures 2014, 73, 171-184, https://doi.org/10.1016/j.spmi.2014.05.023.

25. Ghazi, H.E.; Jorio, A.; Zorkani, I. Pressure-dependent of linear and nonlinear optical properties of (In,Ga)N/GaN spherical QD. Superlattices and Microstructures 2014, 71, 211-216, https://doi.org/10.1016/j.spmi.2014.03.046.

26. Baskoutas, S.; Paspalakis, E.; Terzis, A.F. Excitonic effects in nonlinear optical rectification in small semiparabolic quantum dots. Physica Status Solidi C 2007, 4, 292-294, https://doi.org/10.1002/pssc.200673242.

27. Baskoutas, S.; Paspalakis, E.; Terzis, A.F. Effects of excitons in nonlinear optical rectification in semiparabolic quantum dots. Phys. Rev. B 2006, 74, https://doi.org/10.1103/PhysRevB.74.153306.

28. Çakir, B.; Yakar, Y.; Özmen, A.; Özgür Sezer, M.; Şahin, M. Linear and nonlinear optical absorption coefficients and binding energy of a spherical quantum dot. Superlattices and Microstructures 2010, 47, 556-566, https://doi.org/10.1016/j.spmi.2009.12.002.

29. Kumar, K.M.; Peter, A.J.; Lee, C.W. Optical properties of a hydrogenic impurity in a confined $Z n_{1-}$ ${ }_{x} \mathrm{Cd} d_{x} \mathrm{Se} / \mathrm{ZnSe}$ spherical quantum dot. Superlattices and Microstructures 2012, 51, 184-193, https://doi.org/10.1016/j.spmi.2011.11.012.

30. Özmen, A.; Yakar, Y.; Çakir, B.; Atav, Ü. Computation of the oscillator strength and absorption coefficients for the intersubband transitions of the spherical quantum dot. Optics Commun. 2009, 282, 3999-4004, https://doi.org/10.1016/j.optcom.2009.06.043.

31. Liu, G.; Guo, K.-X.; Hassanabadi, H.; Lu, L. Linear and nonlinear optical properties in a disk-shaped quantum dot with a parabolic potential plus a hyperbolic potential in a static magnetic field. Physica B 2012, 407, 3676-3682, https://doi.org/10.1016/j.physb.2012.05.049.

32. Li, B.; Guo, K.X.; Liu, Z.L.; Zheng, Y.B. Nonlinear optical rectification in parabolic quantum dots in the presence of electric and magnetic fields. Phys. Lett. A 2008, 372, 1337-1340, https://doi.org/10.1016/j.physleta.2007.09.075.

33. Duque, C.A.; Porras-Montenegro, N.; Barticevic, Z.; Pacheco, M.; Oliveira, L.E. Effects of applied magnetic fields and hydrostatic pressure on the optical transitions in self-assembled InAs/GaAs quantum dots. J. Phys.: Condensed Matter 2006, 18.

34. Kirak, M.; Yilmaz, S.; Şahin, M.; Gencaslan, M. The electric field effects on the binding energies and the nonlinear optical properties of a donor impurity in a spherical quantum dot. J. Appl.Phys. 2011, 109, https://doi.org/10.1063/1.3582137.

35. Kirak, M.; Yilmaz, S.; Temizer, Ü. Nonlinear optical rectification and oscillator strength in a spherical quantum dot with parabolic confinement in the presence of the electric field. J. Nanoelectronics and Optoelectronics 2013, 8, 165-169, https://doi.org/10.1166/jno.2013.1447.

36. Hakimyfard, A.; Barseghyan, M.G. Kirakosyan, A.A. Simultaneous effects of pressure and magnetic field on intersubband optical transitions in Pöschl-Teller quantum well. Physica E 2009, 41, 1596-1599, https://doi.org/10.1016/j.physe.2009.05.008.

37. Bahramiyan, H. Electric field and impurity effect on nonlinear optical rectification of a double cone like quantum dot. Optical Materials 2018, 75, 187-195, https://doi.org/10.1016/j.optmat.2017.10.014.

38. Sari, H.; Ungan, F.; Sakiroglu, S.; Yesilgul, U.; Sökmen, I. Impurity-related optical response in cylindrical quantum dots with a $\delta$-doped axial potential under an intense laser field. J. Phys. Chem. Solids 2018, 120, 279-286, https://doi.org/10.1016/j.jpcs.2018.04.038.

39. Khordad, R. Effects of magnetic field and geometrical size on the interband light absorption in a quantum $\begin{array}{llllll}\text { pseudodot System. Solid } & \text { State } & \text { 2010, } & \text { 1253-1256, }\end{array}$ https://doi.org/10.1016/j.solidstatesciences.2010.03.001

40. Sakiroglu, S.; Kilic, D.G.; Yesilgul, U.; Ungan, F.; Kasapoglu, E.; Sari, H.; Sokmen, I. Third-harmonic generation of a laser-driven quantum dot with impurity. Physica $B$ 2018, 539, 101-105, https://doi.org/10.1016/j.physb.2018.04.005.

41. Bahar, M.K.; Ungan, F.; Soylu, A. The optical ranges for the optical properties of two-electron quantum dot immersed in plasmas. Physica E 2019, 114, https://doi.org/10.1016/j.physe.2019.113567.

42. Ungan, F.; Pal, S.; Bahar, M.K.; Mora-Ramos, M.E. Computation of the nonlinear optical properties of ntype asymmetric triple $\delta$-doped GaAs quantum well. Superlattices and Microstructures 2019, 130, 76-86, https://doi.org/10.1016/j.spmi.2019.04.023.

43. Aydin, F.; Sari, H.; Kasapoglu, E.; Sakiroglu, S.; Sokmen, I. Anisotropy dependence of the optical response in an impurity doped quantum dot under intense laser field. Physica $E$ 2019, 114, https://doi.org/10.1016/j.physe.2019.113566.

44. Ghajarpour-Nobandegani, S.; Karimi, M.J. Effects of hydrogenic impurity and external fields on the optical absorption in a ring-shaped elliptical quantum dot. Optical Materials 2018, 82, 75-80, https://doi.org/10.1016/j.optmat.2018.05.045.

45. Kilic, D.G.; Sakiroglu, S.; Sokmen, I. Imurity-related optical properties of a laser-driven quantum dot. Physica E 2018, 102, 50-57, https://doi.org/10.1016/j.physe.2018.04.028.

46. M'zerd, S.; Haouari, M.E.; Talbi, A.; Feddi, E.; Mora-Ramos, M.E. Impact of electron-LO-phonon correction and donor impurity localization on the linear and nonlinear optical properties in spherical 
core/shell semiconductor quantum dots. Journal of Alloys and Compounds 2018, 753, 68-78, https://doi.org/10.1016/j.jallcom.2018.04.185.

47. Yakar, Y.; Çakır, B.; Özmen, A. Dipole and quadrupole polarizabilities and oscillator strengths of spherical quantum dot. Chemical Physics 2018, 513, 213-220, https://doi.org/10.1016/j.chemphys.2018.07.049.

48. Ungan, F.; Mora-Ramos, M.E.; Barseghyan, M.G.; Pérez, L.M.; Laroze, D. Intersubband optical properties of a laser-dressed asymmetric triple quantum well nanostructure. Physica E 2019, 114, https://doi.org/10.1016/j.physe.2019.113647.

49. Mora-Ramos, M.E.; Aouami, A.E.; Feddi, E.; Radu, A.; Restrepo, R.L.; Vinasco, J.A.; Morales, A.L.; Duque, C.A. Donor impurity energy and optical absorption in spherical sector quantum dots. Heliyon 2020, 6, e03194, https://doi.org/10.1016/j.heliyon.2020.e03194.

50. Al, E.B.; Kasapoglu, E.; Sakiroglu, S.; Sari, H.; Sökmen, I.; Duque, C.A. Binding energies and optical absorption of donor impurities in spherical quantum dot under applied magnetic field. Physica E 2020, 119, 114011, https://doi.org/10.1016/j.physe.2020.114011.

51. Linnros, J. Carrier lifetime measurement using free carrier absorption transients. I. Principle and injection dependence. J. Appl. Phys. 1998, 84, 275-283, https://doi.org/10.1063/1.368024.

52. Hilborn, R.C. Einstein coefficients, cross sections, f values, dipole moments, and all that. Am. J. Phys. 1982, 50, 982-986, https://doi.org/10.1119/1.12937.

53. Fedorov, A.V.; Baranov, A.V.; Rukhlenko, I.D.; Masumoto, Y. New many-body mechanism of intraband carrier relaxation in quantum dots embedded in doped heterostructures. Solid State Commun. 2003, 128, 219-223, https://doi.org/10.1016/j.ssc.2003.08.019.

54. Makhlouf, D.; Choubani, M.; Saidi, F.; Maaref, H. Enhancement of transition lifetime, linear and nonlinear optical properties in laterally coupled lens-shaped quantum dots for Tera-Hertz range. Physica E 2018, 103, 87-92, https://doi.org/10.1016/j.physe.2018.05.024.

55. Belevskii, P.A.; Vinoslavskii, M.N.; Poroshin, V.N.; Baidus, N.V.; Zvonkov, B.N. Interband and intraband

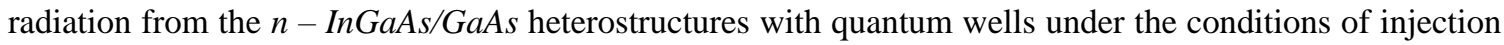
in high lateral electric fields. Physica E 2015, 74, 328-333, https://doi.org/10.1016/j.physe.2015.07.023.

56. Rajashabala, S.; Navaneethakrishnan, K. Effects of dielectric screening and position dependent effective mass on donor binding energies and on diamagnetic susceptibility in a quantum well. Superlattices and Microstructures 2008, 43, 247-261, https://doi.org/10.1016/j.spmi.2007.11.002.

57. Rajashabala, S.; Navaneethakrishnan, K. Effective masses for donor binding energies in quantum well systems. Mod. Phys. Lett. B 2006, 20, 1529-1541, https://doi.org/10.1142/S0217984906011633.

58. Peter, A.J.; Navaneethakrishnan, K. Effects of position-dependent effective mass and dielectric function of a hydrogenic donor in a quantum dot. Physica E 2008, 40, 2747-2751, https://doi.org/10.1016/j.physe.2007.12.025.

59. Khordad, R. Effects of position-dependent effective mass of a hydrogenic donor impurity in a ridge quantum wire. Physica E 2010, 42, 1503-1508, https://doi.org/10.1016/j.physe.2009.12.006.

60. Khordad, R. Effect of position-dependent effective mass on linear and nonlinear optical properties of a cubic quantum dot. Physica B 2011, 406, 3911-3916, https://doi.org/10.1016/j.physb.2011.07.022.

61. Qi, X.H.; Kang, X.J.; Liu, J.J. Effect of a spatially dependent effective mass on the hydrogenic impurity binding energy in a finite parabolic quantum well. Phys. Rev. B 1998, 58, 10578-10582, https://doi.org/10.1103/PhysRevB.58.10578.

62. Peter, A.J. The effect of position-dependent effective mass of hydrogenic impurities in parabolic GaAs/GaAlAs quantum dots in a strong magnetic field. Int. J. Mod. Phys. B 2009, 26, 5109-5118, https://doi.org/10.1142/S0217979209053394.

63. Li, Y.X.; Liu, J.J.; Kang, X.J. The effect of a spatially dependent effective mass on hydrogenic impurity binding energy in a finite parabolic quantum well. J. Appl. Phys. 2000, 88, 2588-2592, https://doi.org/10.1063/1.1286244.

64. Naimi, Y.; Vahedi, J.; Soltani, M.R. Effect of position-dependent effective mass on optical properties of spherical nanostructures. Opt. Quant. Electron. 2015, 47, 2947-2956, https://doi.org/10.1007/s11082-0150183-5.

65. Deng, Z.Y.; Guo, J.K.; Lai, T.R. Impurity states in a spherical GaAs.Ga ${ }_{1-\mathrm{x}} \mathrm{Al}_{\mathrm{x}} \mathrm{As}$ quantum dot: Effects of the spatial variation of dielectric screening. Phys. Rev. B 1994, 50, 5736-5739, https://doi.org/10.1103/physrevb.50.5736.

66. Xie, W. Third-order nonlinear optical susceptibility of a donor in elliptical quantum dots. Superlattices and Microstructures 2013, 53, 49-54, https://doi.org/10.1016/j.spmi.2012.09.009.

67. Xie, W. Optical anisotropy of a donor in ellipsoidal quantum dots. Physica B 2012, 407, 4588-4591, https://doi.org/10.1016/j.physb.2012.08.023.

68. Safarpour, Gh.; Izadi, M.A.; Novzari, M.; Niknam, E.; Moradi, M. Anisotropy effect on the nonlinear optical properties of a three-dimensional quantum dot confined at the center of a cylindrical nano-wire. Physica E 2014, 59, 124-132, https://doi.org/10.1016/j.physe.2014.01.007.

69. Safarpour, G.; Izadi, M.A.; Novzari, M.; Yazdanpanahi, S. Anisotropy effect on the linear and nonlinear optical properties of a laser dressed donor impurity in a GaAs/GaAlAs nanowire superlattice. Superlattices and Microstructures 2014, 75, 936-947, https://doi.org/10.1016/j.spmi.2014.09.018. 
70. Karki, H.D.; Elagöz, S.; Başer, P. Simultaneous effects of hydrostatic pressure and temperature on the binding energy of hydrogenic impurity in cylindrical quantum well wires. Superlattices and Microstructures 2010, 48, 298-304, https://doi.org/10.1016/j.spmi.2010.06.021.

71. Lu, L.; Xie, W.; Shu, Z. Combined effects of hydrostatic pressure and temperature on nonlinear properties of an exciton in a spherical quantum dot under the applied electric field. Physica B 2011, 406, 3735-3740, https://doi.org/10.1016/j.physb.2011.06.081.

72. Yariv, A. Quantum Electronics. 3rd Edn. John Wiley and Sons, Volume 389, New York, 1989.

73. Ditchburn, R.W. Light. 3rd Edn. Academic, New York, 1976. 